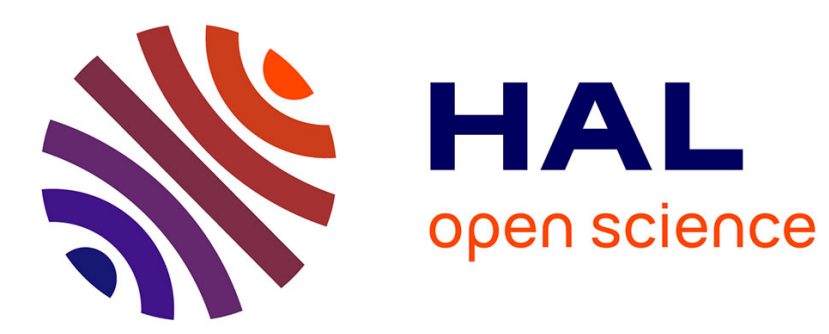

\title{
A Similarity Skyline Approach for Handling Graph Queries - A Preliminary Report
}

\author{
Katia Abbaci, Allel Hadjali, Ludovic Liétard, Daniel Rocacher
}

\section{To cite this version:}

Katia Abbaci, Allel Hadjali, Ludovic Liétard, Daniel Rocacher. A Similarity Skyline Approach for Handling Graph Queries - A Preliminary Report. International Workshop on Graph Data Management Techniques and Applications (GDM'11), in Conjunction with The IEEE International Conference on Data Engineering (ICDE),, Apr 2011, Germany. pp.112-117. hal-00670641

\section{HAL Id: hal-00670641 \\ https://hal.science/hal-00670641}

Submitted on 15 Feb 2012

HAL is a multi-disciplinary open access archive for the deposit and dissemination of scientific research documents, whether they are published or not. The documents may come from teaching and research institutions in France or abroad, or from public or private research centers.
L'archive ouverte pluridisciplinaire HAL, est destinée au dépôt et à la diffusion de documents scientifiques de niveau recherche, publiés ou non, émanant des établissements d'enseignement et de recherche français ou étrangers, des laboratoires publics ou privés. 


\title{
A Similarity Skyline Approach for Handling Graph Queries - A Preliminary Report
}

\author{
Katia Abbaci ${ }^{\# 1}$, Allel Hadjali ${ }^{\# 2}$, Ludovic Liétard ${ }^{* 3}$, Daniel Rocacher ${ }^{* 4}$, \\ \# IRISA/ENSSAT \\ Rue de Kérampont BP 80518 Lannion, France \\ ${ }^{1}$ Katia.Abbaci@enssat.fr \\ ${ }^{2}$ Allel.Hadjali@enssat.fr \\ ${ }^{4}$ Daniel.Rocacher@enssat.fr \\ ${ }^{*}$ IRISA/IUT \\ Rue Edouard Branly BP 30219 Lannion, France \\ ${ }^{3}$ Ludovic.Lietard@univ-rennesl.fr
}

\begin{abstract}
One of the fundamental problems in graph databases is similarity search for graphs of interest. Existing approaches dealing with this problem rely on a single similarity measure between graph structures. In this paper, we suggest an alternative approach allowing for searching similar graphs to a graph query where similarity between graphs is rather modeled by a vector of scalars than a unique scalar. To this end, we introduce the notion of similarity skyline of a graph query defined by the subset of graphs of the target database that are the most similar to the query in a Pareto sense. The idea is to achieve a d-dimensional comparison between graphs in terms of $d$ local distance (or similarity) measures and to retrieve those graphs that are maximally similar in the sense of the Pareto dominance relation. A diversity-based method for refining the retrieval result is proposed as well.
\end{abstract}

\section{INTRODUCTION}

Graphs have become increasingly important in modeling complex structured data in many recent real applications. These applications include Bioinformatics [1], [2], Pattern Recognition [3], XML documents [4], Chemical compounds [5], Social networks [6], etc. All these applications indicate the importance and the broad usage of graph databases. One can broadly classify queries against graph databases into two categories [7]: (1) Graph containment search and (2) Graph similarity search. The former consists of the following two sub-problems: (i) subgraph containment search: given a graph database $D=\left\{g_{1}, g_{2}, \ldots, g_{n}\right\}$ and a graph query $q$, retrieve all graphs $g_{i} \in D$ such that $q$ is a subgraph of $g_{i}$ (i.e., $q \subseteq g_{i}$ ); (ii) supergraph containment search: given a graph database $D=\left\{g_{1}, g_{2}, \ldots, g_{n}\right\}$ and a graph query $q$, retrieve all graph $g_{i} \in D$ such that $q$ is a supergraph of $g_{i}$ (i.e., $q \supseteq g_{i}$ ). Both sub-problems consider the procedure of checking subgraph isomorphism, known to be NP-Complete. Many query processing approaches using indexing techniques have been developed to reduce the search space and then efficiently solve these two sub-problems [8], [9], [10], [11].

As for the second category (i.e., graph similarity search), which consists in retrieving all the graphs of the database that are structurally similar to a given graph query, has emerged as new trend due to the following reasons [12], [13]. Firstly, many real graph datasets are noisy and incomplete in nature, so approximate, rather than exact, graph matching is required. Secondly, many graph applications prefer approximate matching results rather than exact ones as they can provide more information such as what might be missing or spurious in a query or in a graph database. A number of approaches therefore have been proposed to support similarity queries on graph databases, see [14], [15] and [12] in the case of subgraph queries and [16] in the case of supergraph queries. The common point of all those approaches is the fact that they impose a single measure to evaluate graph similarity. However, a graph is a complex structure by nature and involves various basic features. It is then difficult to give a meaningful definition of graph similarity using only a single index.

In this paper, we advocate that for graph similarity to be efficiently assessed, several indices are required. Each index is dedicated to measure a local distance (or similarity) between two graphs pertaining to one aspect in the graph structure. Therefore, graph similarity is now characterized by a vector of local distance measures (where each measure expresses a feature similarity) instead of a single measure. By this way, one can preserve information about similarity on several features when comparing two graphs.

We propose an approach based on the notion of similarity skyline to support graph similarity search. Roughly speaking, the similarity skyline of a graph query is defined by the subset of graphs of the target database that are the most similar to the query in a Pareto sense. The idea is to achieve a $d$ dimensional comparison between graphs in terms of $d$ local distance (or similarity) measures and to retrieve those graphs that are maximally similar in the sense of a defined similaritydominance relation. In summary, we made the following contributions in this paper:

- We introduce the notion of graph compound similarity and then define the similarity-dominance relationship between graphs.

- Based on that relationship, we give a formal definition 
of the graph similarity skyline, i.e., graphs of the target database that are maximally similar to a graph query in a Pareto sense.

- To reduce the resulting skyline (which is often quite large), we propose a method to extract a subset which is as diverse as possible, but with an acceptable size.

The rest of the paper is organized as follows. Section 2 provides some preliminary notions. Related work is discussed in Section 3. Section 4 describes some well-known measures for graph similarity and their semantic properties. In Section 5, we introduce the notion of similarity skyline to support graph similarity queries. Section 6 proposes a detailed example. Section 7 presents a method for refining the retrieval result. Section 8 concludes the paper.

\section{PRELIMINARIES}

\section{A. Reminder About Skyline Queries}

Skyline queries [17] are a popular and powerful paradigm for extracting interesting objects from a multi-dimensional dataset. They rely on Pareto dominance principle which can be defined as follows:

Definition 1. Let $r$ be a set of $d$-dimensional data points and $p=\left(p_{1}, p_{2}, \ldots, p_{d}\right)$ and $q=\left(q_{1}, q_{2}, \ldots, q_{d}\right)$ two points of $r . p$ is said to dominate (in the Pareto sense) $q$ iff on every dimension $p_{i} \leq q_{i}$ (for $1 \leq i \leq d$ ) and on at least one dimension $p_{j}<q_{j}$.

For simplicity and without loss of generality, we assume that the smaller the value $p_{i}$, the better. We say then that $p$ dominates (is preferred to) $q$ and we denote this by $p \succ q$.

Definition 2. The skyline of $r$ is the set of points which are not dominated by any other point.

Skyline queries compute the set of Pareto-optimal tuples in a relation, i.e., those tuples that are not dominated by any other tuple in the same relation.

Example 1. Consider a database containing information about hotels as shown in Table I (where dimension $d=2$ ).

TABLE I

SAMPLE HOTELS

\begin{tabular}{|c|c|c|}
\hline Hotel & Price $(€)$ & Distance $(\mathrm{Km})$ \\
\hline \hline$H_{1}$ & 4.0 & 150 \\
\hline$H_{2}$ & 3.0 & 110 \\
\hline$H_{3}$ & 2.5 & 240 \\
\hline$H_{4}$ & 2.0 & 180 \\
\hline$H_{5}$ & 1.7 & 270 \\
\hline$H_{6}$ & 1.0 & 195 \\
\hline$H_{7}$ & 1.2 & 210 \\
\hline
\end{tabular}

Consider a person who looks for a hotel that is as close as possible to the beach and having a low price. One can check that the resulting skyline $S$ contains $H_{2}, H_{4}$ and $H_{6}$. For instance, $H_{1}$ is dominated by $H_{2}$, and $H_{7}$ by $H_{6}$.

\section{B. Some Basic Definitions}

Definition 3 (Graph). A graph $g$ is defined as a 4-tuple ( $V$, $E, L, l)$ where $V$ is the set of vertices, $E$ is the set of edges, $L$ is the set of labels and $l$ is a labeling function that maps each vertex or edge to a label in $L$.

For ease of presentation, graphs refer here to undirected labeled graphs. Note that different nodes could have the same label and the size of $g$ is defined as $|g|=|E(g)|$ (i.e., the size of a graph is the number of its edges).

Definition 4 (Graph isomorphism). Given two graphs $g=$ $(V, E, L, l)$ and $g^{\prime}=\left(V^{\prime}, E^{\prime}, L^{\prime}, l^{\prime}\right), g$ is isomorphic to $g^{\prime}$ (denoted by $g \approx g^{\prime}$ ) if there exists a bijection $f: V \rightarrow V^{\prime}$, such that

1) $\forall v \in V, f(v) \in V^{\prime}$ and $l(v)=l^{\prime}(f(v))$ and;

2) $\forall(u, v) \in E,(f(u), f(v)) \in E^{\prime}$, and $l(u, v)=$ $l^{\prime}(f(u), f(v))$.

Definition 5 (Subgraph isomorphism). Given two graphs $g$ $=(V, E, L, l)$ and $g^{\prime}=\left(V^{\prime}, E^{\prime}, L^{\prime}, l^{\prime}\right), g$ is subgraph isomorphic to $g$ ' if there exists an injection $f: V \rightarrow V^{\prime}$ such that

1) $\forall v \in V, f(v) \in V^{\prime}$ and $l(v)=l^{\prime}(f(v))$ and;

2) $\forall(u, v) \in E,(f(u), f(v)) \in E^{\prime}$ and $l(u, v)=$ $l^{\prime}(f(u), f(v))$.

Definition 6 (Subgraph v.s. supergraph). Given two graphs $g$ $=(V, E, L, l)$ and $g^{\prime}=\left(V^{\prime}, E^{\prime}, L^{\prime}, l^{\prime}\right), g$ is called a subgraph of $g^{\prime}$ (or $g^{\prime}$ is a supergraph of $g$ ), denoted as $g \subseteq g^{\prime}$ (or $g^{\prime} \supseteq g$ ), if there exists a subgraph isomorphism from $g$ to $g$ '.

Definition 7 (Maximum common subgraph, mcs). Given two graphs $g_{1}$ and $g_{2}$, the maximum common subgraph of $g_{1}$ and $g_{2}$ is the largest (i.e., the maximum number of selected vertices) connected subgraph of $g_{1}$ that is subgraph isomorphic to $g_{2}$, denoted as $g^{\prime}=\operatorname{mcs}\left(g_{1}, g_{2}\right)$.

\section{RELATED WORK}

Our proposal can be related to the works in the areas of skyline queries and similarity queries on graph databases.

Skyline queries. They have received a lot of attention over the recent years. Several research efforts have been made to develop efficient algorithms and to introduce different variants for skyline queries [18], [19], [20], [21]. Up to our knowledge, no work related to skyline queries exists in a graph data context, except the recent work by Zou et al. [22] where dynamic skyline queries in a large graph have been studied. In our case, a different kind of skyline (i.e., similarity skyline) over a set of graphs (rather than a single large graph) is investigated.

Similarity queries. Similarity search of graphs is a vital operation in many recent applications. As indicated in Section I, this kind of graph search is conducted thanks to similarity queries that aim at finding graphs in the target database that are similar, but not necessarily isomorphic, to a given graph query. A number of approaches have been developed to support similarity queries. Grafil [14] performs substructure similarity search in a large scale graph database. It returns all the graphs of the database that approximately contain the graph query. C-Tree [15] is another tool for subgraph similarity search which focuses on the edit distance between the query and its candidate matches. Tale [12], unlike most previous graph matching tools which treat every node in a 
graph equally, proposes an innovative matching technique that distinguishes nodes by their importance in the graph structure. This technique first matches the important nodes of a graph query, and then progressively extends these matches. Recently, Shang et al. [16] have proposed a technique to deal with supergraph queries where the notion of maximum common subgraph plays a key role. The problem of interest is converted into a $\sigma$-missing subgraph detection problem, where $\sigma$ is the error tolerance threshold. All the graphs of the queried database such that the mcs-based distance measure to the graph query considered is less than $\sigma$, are returned as answers. Both $C$-Tree and Tale rely on the edit distance to measure similarity between graphs whereas the works done in [14] and [16] use the notion of maximum common subgraph for computing that similarity.

As can be seen all the approaches that support similarity queries on graph data make use of a unique index to measure similarity between two graphs. So doing, similarity between two graph structures is not wholly captured since some similarities pertaining to some features of graph are missed. This is mainly due to the fact that each index of graph similarity can be seen as a local measure that expresses only a resemblance w.r.t. one aspect in a graph structure (see Section IV). Compared with the above work, our approach, on the one hand, relies on a compound similarity measure between graphs and, on the other hand, returns a set of similarity dominant graphs in a Pareto sense to answer a graph query.

\section{Graph Similarity Measures: Some Semantic PROPERTIES}

Several models have been proposed [23], [24], [25] to measure the similarity (or distance) between two graphs. Hereafter, we present the most widely accepted measures to determine similarities between graphs ${ }^{1}$.

\section{A. Graph Edit Distance}

Graph edit distance [15], [23] is based on graph edit operations needed to transforme one graph to another. Generally, the set of edit operations considered includes: insertion or deletion of a vertex/edge and relabeling of a vertex/edge. Each edit operation is associated with a cost (a non-negative real number) according to the amount of distortion that it introduces in the transformation. Let $e_{-} o p$ be an edit operation and $c\left(e_{-} o p\right)$ its cost. The cost of a sequence of edit operations, $s=\left(e_{-} o p_{1}, \ldots, e_{-} o p_{n}\right)$ is given by

$$
c(s)=\sum_{i=1}^{n} c\left(e_{-} o p_{i}\right) .
$$

The choice of elementary edit operations and their cost represent a difficult task in practice. The cost of a transformation of an element to another can be regarded as a distance function between the two elements. We assume here a uniform distance measure: the distance between two vertices/edges is 1 if they have different labels; otherwise it is 0 .

\footnotetext{
${ }^{1}$ Due to space limitation, the computational complexity analysis of each measure is not addressed here.
}

Definition 8 (Graph edit distance). The edit distance between two graphs $g_{1}$ and $g_{2}$ is equal to the minimum cost, taken over all sequences of edit operations, that transform $g_{1}$ into $g_{2}$, i.e.,

$$
\operatorname{Dist}_{E d}\left(g_{1}, g_{2}\right)=\min _{s \in E_{-} o p} c(s)
$$

where $E \_o p$ denotes the set of all sequences of edit operations that transform $g_{1}$ into $g_{2}$.

The smaller $\operatorname{Dist}_{E d}\left(g_{1}, g_{2}\right)$, the more similar the two graphs. One can easily check that the edit distance between isomorphic graphs is zero.

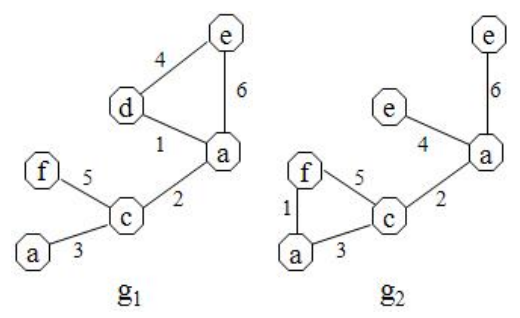

Fig. 1. Examples of labeled graph

Example 2. Let us consider the labeled graphs of Fig.1. The sequence of edit operations those are necessary for transforming $g_{1}$ into $g_{2}$ is: (i) one edge deletion, (ii) one edge relabeling, (iii) one vertex relabeling, (iv) one edge insertion. By considering uniform distance measures, one can check that this sequence is the best one (i.e., the shortest). So, Dist $_{E d}\left(g_{1}, g_{2}\right)=4$.

It is worth noticing that, in a graph database querying context, this distance measure provides information on features that both a graph of the target database and the graph query at hand disagree.

\section{B. Mcs-Based Distance}

Bunke et al. [24] have developed another kind of measure for graph similarity. It is based on the maximum common subgraph (mcs).

Definition 9 (Similarity based on the mcs). Given two graphs $g_{1}$ and $g_{2}$, the graph similarity based on the mcs is defined as,

$$
\operatorname{Sim}_{M c s}\left(g_{1}, g_{2}\right)=\frac{\left|\operatorname{mcs}\left(g_{1}, g_{2}\right)\right|}{\max \left(\left|g_{1}\right|,\left|g_{2}\right|\right)},
$$

where $\left|m c s\left(g_{1}, g_{2}\right)\right|$ denotes the number of edges in $\operatorname{mcs}\left(g_{1}, g_{2}\right)$.

Clearly, the larger the mcs of two graphs the greater their similarity. The measure $\operatorname{Sim}_{M c s}$ is normalized (i.e., $0 \leq$ $\left.\operatorname{Sim}_{M c s}\left(g_{1}, g_{2}\right) \leq 1\right)$ since $\left|\operatorname{mcs}\left(g_{1}, g_{2}\right)\right| \leq \max \left(\left|g_{1}\right|,\left|g_{2}\right|\right)$. Now, the graph distance measure, Dist ${ }_{M c s}$, derived from Sim $_{M c s}$ writes:

$$
\operatorname{Dist}_{M c s}\left(g_{1}, g_{2}\right)=1-\operatorname{Sim}_{M c s}\left(g_{1}, g_{2}\right)
$$

Such a measure is proved to be a metric in [24] and leads to a distance in $[0,1]$.

The major advantage of the mcs-based approach is the fact that it does not require the use of any cost function, thereby avoiding the main drawback of edit-distance-based approach. 
Example 3. Let us come back to Example 2. The mcsbased distance measure between $g_{1}$ and $g_{2}$ is calculated as follows. First, the $\operatorname{mcs}\left(g_{1}, g_{2}\right)$ is identified, see Fig. 2. Then, by applying (2), we obtain

$$
\operatorname{Dist}_{M c s}\left(g_{1}, g_{2}\right)=1-\frac{\left|m c s\left(g_{1}, g_{2}\right)\right|}{\max \left(\left|g_{1}\right|,\left|g_{2}\right|\right)}=0.33
$$$$
\text { where }\left|\operatorname{mcs}\left(g_{1}, g_{2}\right)\right|=4 \text { and } \max \left(\left|g_{1}\right|,\left|g_{2}\right|\right)=6 \text {. }
$$

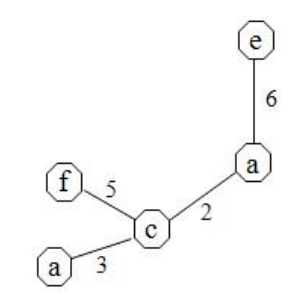

Fig. 2. Mcs of $g_{1}$ and $g_{2}$

From a database querying point of view, this kind of similarity conveys information on the amount of features that both a graph of the queried database and a graph query agree.

\section{Gu-Based Distance}

Graph union $(\mathrm{Gu})$-based distance measure, introduced by Wallis et al. [25], is based on the idea of graph union. Graph union (rather than the larger of two graphs) is used to model the size of the problem.

Definition 10 (Gu-based similarity). Given two graphs $g_{1}$ and $g_{2}$, the graph similarity based on graph union is defined as,

$$
\operatorname{Sim}_{G u}\left(g_{1}, g_{2}\right)=\frac{\left|m c s\left(g_{1}, g_{2}\right)\right|}{\left|g_{1}\right|+\left|g_{2}\right|-\left|m c s\left(g_{1}, g_{2}\right)\right|},
$$

where the denominator represents the size of union of the two graphs in the set theoretic sense ${ }^{2}$.

This similarity measure is also normalized and its behaviour is somewhat close to $\operatorname{Sim}_{M c s}$. It is easy to see that $\operatorname{Sim}_{G u}\left(g_{1}, g_{2}\right) \leq \operatorname{Sim}_{M c s}\left(g_{1}, g_{2}\right)$ holds as well (which means that $\operatorname{Sim}_{G u}$ is a stronger measure than $\left.\operatorname{Sim}_{M c s}\right)$. The use of graph union [25] is motivated by the fact that changes in the size of the smallest graph that keep $m c s\left(g_{1}, g_{2}\right)$ constant are not taken into account in $\operatorname{Sim}_{M c s}\left(g_{1}, g_{2}\right)$ whereas the measure $\operatorname{Sim}_{G u}\left(g_{1}, g_{2}\right)$ does take this variation into account.

The graph distance measure derived from $\operatorname{Sim}_{G u}$ can be written as:

$$
\operatorname{Dist}_{G u}\left(g_{1}, g_{2}\right)=1-\operatorname{Sim}_{G u}\left(g_{1}, g_{2}\right)
$$

It was also proved to be a metric and gives values in $[0,1]$.

Example 4. Let us again consider the graphs provided in Example 2. Using (3), the Gu-based distance measure between $g_{1}$ and $g_{2}$ is

$$
\operatorname{Dist}_{G u}\left(g_{1}, g_{2}\right)=1-\frac{\left|m c s\left(g_{1}, g_{2}\right)\right|}{\left|g_{1}\right|+\left|g_{2}\right|-\left|m c s\left(g_{1}, g_{2}\right)\right|}=0.50 \text {, }
$$

where $\left|m c s\left(g_{1}, g_{2}\right)\right|=4$ (see Example 3) and $\left|g_{1}\right|=\left|g_{2}\right|=6$.

In a database querying context, this type of similarity gives also information about the number of agreements between a graph of the queried database and a graph query.

\footnotetext{
${ }^{2}$ This similarity measure looks like the Jaccard index used to measure similarity between two sets $A$ and $B$, i.e., $J(A, B)=|A \cap B| /|A \cup B|$.
}

\section{GRAPH Similarity SKYLINE}

This section is devoted to define the notion of similarity skyline for supporting graph similarity search without the need for specifying a global similarity measure between graph structures.

From now on, we assume that graph similarity is a compound notion, i.e., in order to assess similarity between graphs we consider a vector of distance measures. Each measure can be regarded as a local similarity expressing the extent to which two graphs are similar w.r.t. some features or aspects.

Definition 11 (Graph Compound Similarity, GCS). Let $g$ and $g$ ' be two graphs, a graph compound similarity between $g$ and $g$ ' is a vector of local distance measures denoted by

$$
G C S\left(g, g^{\prime}\right)=\left(\operatorname{Dist}_{1}\left(g, g^{\prime}\right), \operatorname{Dist}_{2}\left(g, g^{\prime}\right), \ldots, \operatorname{Dist}_{d}\left(g, g^{\prime}\right)\right) \text {, }
$$

where $\operatorname{Dist}_{i}\left(g, g^{\prime}\right)$, for $\mathrm{i}=1, \ldots, \mathrm{d}$, stands for a local graph distance measure.

Let now $D=\left\{g_{1}, g_{2}, \ldots, g_{n}\right\}$ be a graph database and $q$ a graph similarity query (i.e., this means that the user is interested in graphs of $D$ that are the most similar to $q$ ). Since a global similarity between graphs is not available, the idea is to proceed with a d-dimensional comparison between graphs in terms of $d$ (local) distance measures to retrieve graphs that are maximally similar in the sense of the following similaritydominance relation.

Definition 12 (Similarity-dominance relation). Given a graph query $q$ and two graphs $g$ and $g$ ', we say that $g$ ' is similaritydominated by $g$ in the context of $q$, denoted by $g \succ_{q} g^{\prime}$, iff the following two statements hold:

1) $\forall i \in\{1, \ldots, d\}, \operatorname{Dist}_{i}(g, q) \leq \operatorname{Dist}_{i}\left(g^{\prime}, q\right)$,

2) $\exists k \in\{1, \ldots, d\}, \operatorname{Dist}_{k}(g, q)<\operatorname{Dist}_{k}\left(g^{\prime}, q\right)$.

Roughly speaking, the relation $g \succ_{q} g^{\prime}$ holds if $g$ is not less similar to $q$ than $g$ ' in all dimensions and (strictly) more similar to $q$ than $g$ ' in at least one dimension. One can observe that $g$ is potentially more interesting than $g$ ' as a retrieval graph. Therefore, the set of graphs that are the most similar to $q$ are those that are not dominated (in the sense of Definition 12). Such graphs, called Pareto-optimal graphs, represent what we denote by the graph similarity skyline (GSS):

$$
G S S(D, q)=\left\{g \in D \mid \nexists g^{\prime} \in D, g^{\prime} \succ_{q} g\right\}
$$

where $g^{\prime} \succ_{q} g$ means that $g$ is similarity-dominated by $g$ '.

To illustrate the above approach, we provide in the next section an example where $d=3 . G C S(g, q)$ is then a vector of three components expressed by the local distance measures described in Section IV, i.e.,

$$
G C S(g, q)=\left(\operatorname{Dist}_{E d}(g, q), \operatorname{Dist}_{M c s}(g, q), \operatorname{Dist}_{G u}(g, q)\right) \text {. }
$$

\section{AN ILlustrative EXAMPLE}

Let $D=\left\{g_{1}, g_{2}, g_{3}, g_{4}, g_{5}, g_{6}, g_{7}\right\}$ be a graph database and $q$ a graph similarity query, as shown in Fig. 3. In order to provide the most interesting answers to $q$, one can compute the graph similarity skyline $\operatorname{GSS}(D, q)$. It is easy to see that $\left|g_{1}\right|=6,\left|g_{2}\right|=7,\left|g_{3}\right|=7,\left|g_{4}\right|=6,\left|g_{5}\right|=8,\left|g_{6}\right|=9,\left|g_{7}\right|=$ 


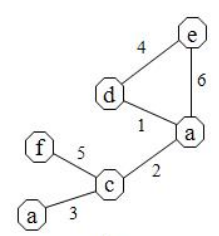

$\mathrm{g}_{1}$

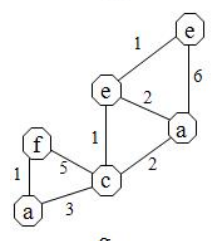

$\mathrm{g}_{5}$

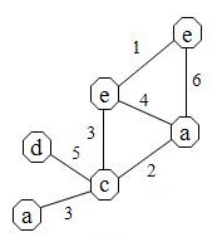

$\mathrm{g}_{2}$

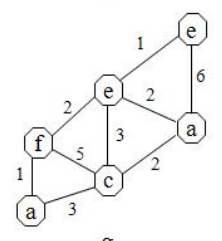

$\mathrm{g}_{6}$

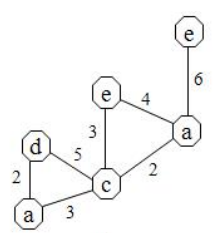

$\mathrm{g}_{3}$

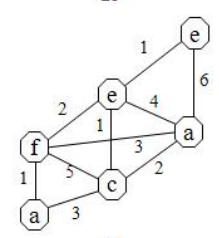

$\mathrm{g}_{7}$

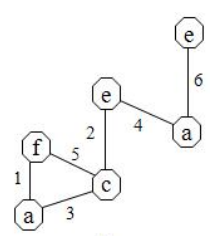

$\mathrm{g}_{4}$

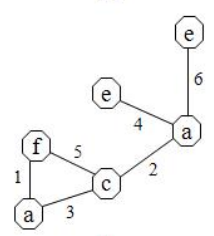

q
Fig. 3. The graph database $D$ and the graph query $q$

10 and $|q|=6$. Table 2 summarizes the values of $\left|\operatorname{mcs}\left(g_{i}, q\right)\right|$, for $\mathrm{i}=1, \ldots, 7$.

TABLE II

INFORMATION ABOUT $\left|M c s\left(g_{i}, q\right)\right|$

\begin{tabular}{|c|c|}
\hline & $\left|\operatorname{Mcs}\left(g_{i}, q\right)\right|$ \\
\hline \hline$\left(g_{1}, q\right)$ & 4 \\
\hline$\left(g_{2}, q\right)$ & 4 \\
\hline$\left(g_{3}, q\right)$ & 4 \\
\hline$\left(g_{4}, q\right)$ & 3 \\
\hline$\left(g_{5}, q\right)$ & 5 \\
\hline$\left(g_{6}, q\right)$ & 5 \\
\hline$\left(g_{7}, q\right)$ & 6 \\
\hline
\end{tabular}

TABLE III

DistanCE MEASURES

\begin{tabular}{|c|c|c|c|}
\hline & Dist $_{E d}\left(g_{i}, q\right)$ & Dist $_{M c s}\left(g_{i}, q\right)$ & Dist $_{G u}\left(g_{i}, q\right)$ \\
\hline$\left(g_{1}, \mathbf{q}\right)$ & $\mathbf{4}$ & $\mathbf{0 . 3 3}$ & $\mathbf{0 . 5 0}$ \\
\hline$\left(g_{2}, \mathbf{q}\right)$ & 4 & 0.43 & 0.56 \\
\hline$\left(g_{3}, \mathbf{q}\right)$ & 3 & 0.43 & 0.56 \\
\hline$\left(g_{4}, \mathbf{q}\right)$ & $\mathbf{2}$ & $\mathbf{0 . 5 0}$ & $\mathbf{0 . 6 7}$ \\
\hline$\left(g_{5}, \mathbf{q}\right)$ & $\mathbf{3}$ & $\mathbf{0 . 3 8}$ & $\mathbf{0 . 4 4}$ \\
\hline$\left(g_{6}, \mathbf{q}\right)$ & 4 & 0.44 & 0.50 \\
\hline$\left(g_{7}, \mathbf{q}\right)$ & $\mathbf{4}$ & $\mathbf{0 . 4 0}$ & $\mathbf{0 . 4 0}$ \\
\hline
\end{tabular}

Now the graph similarity vectors $G C S\left(g_{i}, q\right)$, for $\mathrm{i}=1, \ldots, 7$, are shown in Table III. By applying (4), the set of Pareto optimal graphs, i.e. the graph similarity skyline, is given by $G S S(D, q)=\left\{g_{1}, g_{4}, g_{5}, g_{7}\right\}$.

One can easily check that $g_{2}$ (resp. $\left.g_{3}\right) \notin G S S(D, q)$ since it is dominated by $g_{7}$ (resp. $g_{5}$ ) and $g_{6} \notin G S S(D, q)$ since it is dominated by $g_{1}$. Thus, the graphs of $D$ that are maximally similar to $q$ are $g_{1}, g_{4}, g_{5}$ and $g_{7}$. Indeed,

- Graph $g_{1}$ is the most interesting w.r.t. the measure Dist $_{M c s}$. This is due to the two following reasons: i) $g_{1}$ satisfies a maximum number of features required by $q$ than other graphs with the same size; ii) $g_{1}$ and $q$ are of the same size. But, $g_{1}$ is the less interesting w.r.t. to superfluous and missing features.
- Graph $g_{4}$ is the best w.r.t. the measure Dist ${ }_{E d}$. This means that it is the most interesting w.r.t. to the numbers of disagreements with $q$. On the other hand, $g_{4}$ is much less satisfactory w.r.t. to the agreements with $q$ in the sense of the mcs notion.

- Graph $g_{7}$ is the most interesting w.r.t. the measure Dist $_{G u}$. This is due to the fact that $g_{7} \supset q$. But, it is the less interesting w.r.t. a superfluous feature-based criterion.

- Graph $g_{5}$ may be a good compromise between the three measures Dist $_{E d}$, Dist $_{M c s}$ and Dist $_{G u}$.

Let us now take a look at the results obtained when using only a single similarity measure between graphs. If we are interested in the best $k(=3)$ answers, $g_{3}$ is then returned for instance by the edit-distance-based approach as answer to the user, but with the skyline-based approach $g_{3}$ is not returned as answer since $g_{5}$ does better than it.

\section{REFINING GRAPH SIMILARITY SKYLINE}

One of the problems that may arise when computing the set GSS (and a skyline in general) is its size which is often quite large. From a user point of view, it is very desirable to have a suitable criterion to select a small interesting subset of graphs of the skyline GSS. One solution is to use the criterion of diversity [26] to select a subset of graphs which is as diverse as possible and then provide the user with a picture of the whole set $G S S$.

Let $S$ be a subset of GSS. The diversity of $S$ means that the graphs it includes should be dissimilar amongst each other. The goal is to extract from GSS a subset $\mathbb{S}$ of size $k$ (a user-defined parameter) with a maximal diversity. Adapted from [27], the proposed approach defines the diversity of $S$ $(\subseteq G S S)$ of size $k$ by a vector $\operatorname{Div}(S)=\left(v_{1}, v_{2}, v_{3}\right)$ such as

$$
v_{i}=\min \left\{\operatorname{Dist}_{i}\left(g, g^{\prime}\right) \mid g, g^{\prime} \in S\right\},
$$

where Dist $_{1}=D_{i s t}-E d$ (the normalized version of the

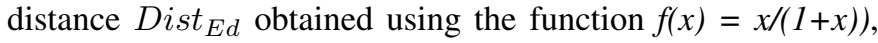

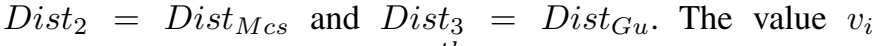
expresses the diversity in the $i^{t h}$ dimension of the subset $S$.

To identify the subset $\mathbb{S}$ of interest, we consider all subsets $S \subseteq G S S$ with $|S|=k$ (i.e., the size of $S$ is $k$ ) as candidates and apply the following steps:

Step 1. For each dimension $i(\mathrm{i}=1, \ldots, 3)$, rank-order all candidates subsets $S$ in decreasing way according to their diversity $v_{i}$ in that dimension. Let $\operatorname{rank}_{i}(S)$ be the rank of $S$ w.r.t. $i^{\text {th }}$ dimension. Rank value 1 means the best diversity value and rank value $M$ means the worst diversity value ( $M$ is the number of subsets of size $k$ of the set $G S S$ ).

Step 2. Evaluate a candidate $S$ by:

$$
\operatorname{val}(S)=\sum_{i=1, \ldots, 3} \operatorname{rank}_{i}(S) .
$$

The subset that minimizes this criterion (i.e., minimizes the sum of its positions in all ranks) is considered as a subset with a maximal diversity. So, $\mathbb{S}$ is characterized by

$$
\operatorname{val}(\mathbb{S})=\min _{S} \operatorname{val}(S)
$$


where $S \subseteq G S S$ and $|S|=k$.

Example 5. Let us come back to the example given in section VI, where $G S S(D, q)=\left\{g_{1}, g_{4}, g_{5}, g_{7}\right\}$. Assume now that the user is interested in the best $k(=2)$ graphs w.r.t. the diversity criterion. One can easily check that the set of all candidates contains 6 subsets of size $k$, see Table IV.

TABLE IV

CANDIDATES WITH THEIR DIVERSITY

\begin{tabular}{|c|c|c|c|}
\hline & $v_{1}$ & $v_{2}$ & $v_{3}$ \\
\hline$S_{1}=\left\{g_{1}, g_{4}\right\}$ & 0.86 & 0.67 & 0.80 \\
\hline$S_{2}=\left\{g_{1}, g_{5}\right\}$ & 0.83 & 0.50 & 0.60 \\
\hline$S_{3}=\left\{g_{1}, g_{7}\right\}$ & 0.87 & 0.60 & 0.67 \\
\hline$S_{4}=\left\{g_{4}, g_{5}\right\}$ & 0.80 & 0.62 & 0.73 \\
\hline$S_{5}=\left\{g_{4}, g_{7}\right\}$ & 0.83 & 0.70 & 0.77 \\
\hline$S_{6}=\left\{g_{5}, g_{7}\right\}$ & 0.75 & 0.50 & 0.61 \\
\hline
\end{tabular}

Now, steps 1 and 2 lead to the results depicted in Table V.

TABLE V

Evaluation of All CANDidates.

(a) Ranks $\left(r_{i}=\operatorname{rank}_{i}\right)$.
\begin{tabular}{|c|c|c|c|}
\hline & $r_{1}$ & $r_{2}$ & $r_{3}$ \\
\hline \hline$S_{1}$ & 2 & 2 & 1 \\
\hline$S_{2}$ & 3 & 5 & 6 \\
\hline$S_{3}$ & 1 & 4 & 4 \\
\hline$S_{4}$ & 4 & 3 & 3 \\
\hline$S_{5}$ & 3 & 1 & 2 \\
\hline$S_{6}$ & 5 & 5 & 5 \\
\hline
\end{tabular}

\begin{tabular}{|c|c|}
\hline & (b) $\operatorname{Val}\left(S_{i}\right)$. \\
\hline & $\sum_{i=1, \ldots, 3} r_{i}$ \\
\hline \hline$S_{1}$ & 5 \\
\hline$S_{2}$ & 14 \\
\hline$S_{3}$ & 9 \\
\hline$S_{4}$ & 10 \\
\hline$S_{5}$ & 6 \\
\hline$S_{6}$ & 15 \\
\hline
\end{tabular}

From Table V-(b), one can easily see that $\operatorname{val}\left(S_{1}\right)$ is the minimal value. So, $\mathbb{S}=S_{1}=\left\{g_{1}, g_{4}\right\}$.

\section{CONCLUSION}

In this paper, we have proposed an alternative approach to support graph similarity search. The key concept of this approach is the notion of graph similarity skyline we introduced. This kind of skyline allows retrieving all graphs of the queried database that are not dominated in the sense of the similarity-dominance relation defined. Namely, graphs those are maximally-similar to the graph query at hand. Each answer graph is provided to the user with a vector of scores showing different similarities pertaining to different features. We have also shown how to select a maximally diverse subset of a graph similarity skyline.

We plan to conduct some experiments on real-life data to demonstrate the effectiveness and efficiency of the approach. To this end, a system implementing it is underway.

\section{ACKNOWLEDGMENTS}

This work was supported in part by the National Agency for Research under project AOC on the reference ANR-08CORD-009, and Brittany region.

\section{REFERENCES}

[1] Y. Tian, R. McEachin, C. Santos, D. J. States, and J. M. Patel, "Saga: a subgaph matching tool for biological graphs," Bioinformatics, vol. 23(2), pp. 232-239, 2007.

[2] H. Hu, Y. Hang, J. Han, and X. Zhou, "Mining coherent dense subgraphs across massive biological network for fonctional discovery," Bioinformatics, vol. 1(1), pp. 1-9, 2005.

[3] D. Conte, P. Foggia, C. Sansone, and M. Vento, "Thirty years of graph matching in pattern recognition," Inter. J. of Pattern Recogn. and Art. Intell., vol. 18 (3), pp. 265-298, 2004.

[4] N. Zhang, T. Ozsu, I. Ilyas, and A. Aboulnaga, "Fix: eature-based indexing technique for xml documents," in Proc. of VLDB, 2006, pp. 259-270.

[5] S. Klinger and J. Austin, "Chemical similarity searching using a neural graph matcher," in Proc. of ESANN, 2005, pp. 479-484.

[6] D. Cai, Z. Shao, X. He, X. Yan, and J. Han, "Community mining from multirelational networks," in Proc. of PPKDD, 2005, pp. 445-452.

[7] Z. Zeng, A. Tung, J. Wang, J. Feng, and L. Zhou, "Comparing stars: On approximating graph edit distance," in Proc. of VLDB, 2009, pp. 25-36.

[8] C. Chen, X. Yan, P. S. Yu, J. Han, D.-Q. Zhang, and X. Gu, "Towards Graph Containment Search and Indexing," in Proc. of VLDB, Vienna, Austria, sept 23-27 2007, pp. 926-937.

[9] X. Yan, P. S. Yu, and J. Han, "Graph indexing: A frequent structurebased approach," in Proc. of ACM SIGMOD, 2004, pp. 335-346.

[10] S. Zhang, J. Z. Li, H. Gao, and Z. Zou, "A novel approach for efficient supergraph query processing on graph databases," in Proc. of EDBT, march 24-26 2009, pp. 204-215.

[11] S. Zhang, M. Hu, and J. Yang, "Treepi: A novel graph indexing method," in Proc. of ICDE, 2007, pp. 966-975.

[12] Y. Tian and J. M. Patel, "Tale : A tool for approximate large graph matching," in Proc. of ICDE, Cancun, Mexico, 2008, pp. 963-972.

[13] E. Petrakis and C. Faloutsos, "Similarity searching in medical image databases," Proc. of TKDE, vol. 9 (3), pp. 435 -447, May 1997.

[14] X. Yan, P. S. Yu, and J. Han, "Substructure similarity search in graph databases," in Proc. of ACM SIGMOD, 2005, pp. 766-777.

[15] H. He and A. K. Singh, "Closure-tree: An index structure for graph queries," in Proc. of ICDE, 2006, pp. 38-52.

[16] H. Shang, K. Zhu, X. Lin, Y. Zhang, and R. Ichise, "Similarity search on supergraph containment," in Proc. of ICDE, march 1-6 2010, pp. 637-648.

[17] S. Borzsonyi, D. Kossmann, and K. Stocker, "The skyline operator," in Proc. of ICDE, 2001, pp. 421-430.

[18] J. Pei, B. Jiang, X. Lin, and Y. Yuan, "Probabilistic skylines on uncertain data," in Proc. of VLDB, 2007, pp. 15-26.

[19] M. L. Yiu and N. Mamoulis, "Efficient processing of top-k dominating queries on multi-dimensional data," in Proc. of VLDB, 2007, pp. 483494.

[20] M. E. Khalefa, M. F. Mokbel, and J. J. Levandoski, "Skyline query processing for incomplete data," in Proc. of ICDE, 2008, pp. 556-565.

[21] A. Hadjali, O. Pivert, and H. Prade, "Possibilistic contextual skylines with incomplete preferences," in Proc. of SoCPaR, Cergy Pontoise, Paris, France, December 07-10, 2010.

[22] L. Zou, L. Chen, M. T. Ozsu, and D. Zhao, "Dynamic skyline queries in large graphs," in Proc. of DASFAA, 2010, pp. 62-78.

[23] H. Bunke, "On a relation between graph edit distance and maximum common subgraph," Pattern Recogn. Letters, vol. 18 (9), pp. 689-697, August 1997.

[24] H. Bunke and K. Shearer, "A graph distance metric based on the maximal common subgraph," Pattern Recogn. Letters, vol. 19 (3-4), pp. 255-259, March 1998.

[25] W. D. Wallis, P. Shoubridge, M. Kraetz, and D. Ray, "Graph distances using graph union," Pattern Recogn. Letters, vol. 22 (6-7), pp. 701-704, May 2001.

[26] D. McSherry, "Diversity-conscious retrieval," in Proc. of ECCBR. Springer-Verlag, 2002, pp. 219-233.

[27] S. Kukkonen and J. Lampinen, "Ranking-dominance and many-objective optimization," in IEEE Congress on Evolutionary Computation, 2007, pp. 3983-3990. 